\title{
Recurrent urinary tract infection and risk of bladder cancer in the Nijmegen bladder cancer study
}

\author{
S H Vermeulen ${ }^{*}$, $\mathrm{N} \mathrm{Hanum}^{2}$, A J Grotenhuis ${ }^{2}$, G Castaño-Vinyals ${ }^{3}$, A G van der Heijden ${ }^{4}, \mathrm{~K} \mathrm{~K} \mathrm{Aben}{ }^{2,5}$, \\ I U Mysorekar ${ }^{6}$ and L A Kiemeney ${ }^{7}$
}

${ }^{1}$ Department for Health Evidence \& Department of Human Genetics, Radboud University Medical Center, PO Box 9101, 6500 HB Nijmegen, The Netherlands; ${ }^{2}$ Department for Health Evidence, Radboud University Medical Center, PO Box 9101,6500 HB Nijmegen, The Netherlands; ${ }^{3}$ Centre for Research in Environmental Epidemiology (CREAL), Doctor Aiguader 88, E-08003 Barcelona, Spain; ${ }^{4}$ Department of Urology, Radboud University Medical Center, PO Box 9101, 6500 HB Nijmegen, The Netherlands; ${ }^{5}$ Comprehensive Cancer Centre the Netherlands, 3511 GD Utrecht, The Netherlands; ${ }^{6}$ Departments of Obstetrics \& Gynecology, Pathology \& Immunology, Washington University School of Medicine, 660S. Euclid Avenue, St. Louis MO 63110, USA and ${ }^{7}$ Department for Health Evidence \& Department of Urology, Radboud University Medical Center, PO Box 9101,6500 HB Nijmegen, The Netherlands

Background: Controversy exists on whether urinary tract infection (UTI) is a risk factor for urinary bladder cancer (UBC). Here, the association is investigated using data from one of the largest bladder cancer case-control studies worldwide.

Methods: Information on (i) history and age at onset of regular cystitis ('regular low-UTI') and (ii) number and age at onset of UTI treated with antibiotics ('UTI-ab') from 1809 UBC patients and 4370 controls was analysed. Odds ratios (ORs) and 95\% confidence intervals (Cl) adjusted for age, education, smoking, and use of aspirin/ibuprofen were generated, for men and women separately.

Results: Regular low-UTI was associated with an increased UBC risk (men: OR (95\% CI) 6.6 (4.2-11); women: 2.7 (2.0-3.5)), with stronger effects in muscle-invasive UBC. Statistically significant decreased risks (ORs $\sim 0.65$ ) were observed for up to five UTI-ab, specifically in those who (had) smoked and experienced UTI-ab at a younger age. In women, UTI experienced after menopause was associated with a higher UBC risk, irrespective of the number of episodes.

Conclusions: Regular cystitis is positively associated with UBC risk. In contrast, a limited number of episodes of UTI treated with antibiotics is associated with decreased UBC risk, but not in never-smokers and postmenopausal women.

Urinary tract infections (UTIs) refer to the presence of microbial pathogens in the urethra or bladder (lower urinary tract), or ureter and pelvis of the kidney (upper urinary tract). In men, the prostate may be involved as well. UTIs are among the most prevalent infectious diseases with varying frequencies among sexes and age categories. About $80 \%$ of UTIs occur in women; it is estimated that $40-50 \%$ of females experience at least one symptomatic UTI and that half of them will experience a recurrence within 1 year (Foxman, 2010; Salvatore et al, 2011). The majority of UTIs is caused by $E$. coli and manifests as cystitis. Risk factors for (recurrent) UTI include obstruction of the urinary tract, use of a bladder catheter, a suppressed immune system, oestrogen deficiency, a genetic predisposition, and sexual intercourse, and may differ between men and pre- and postmenopausal women (Foxman et al, 2000; Nicolle, 2002; Foxman, 2010; Salvatore et al, 2011).

There is strong evidence linking infection of the bladder by Schistosoma haematobium, endemic in certain low- and middle- 
income countries but rare in high-income countries, and by spinal cord injury-related catheter use to squamous cell carcinoma of the bladder (IARC Working Group on the Evaluation of Carcinogenic Risks to Humans, 1994; Hess et al, 2003). However, whether common, recurrent UTIs are involved in the aetiology of urothelial carcinoma of the bladder remains unclear. Several epidemiological studies, most from the second half of the twentieth century and conducted in small populations, have investigated the association between episodes of (lower) UTI and the risk of urinary bladder cancer (UBC). The majority of these studies report increased UBC risks for any vs no UTI, in both men and women (Wynder et al, 1963; Dunham et al, 1968; Howe et al, 1980; Kantor et al, 1984; Claude et al, 1986; La Vecchia et al, 1991; Kunze et al, 1992; Sturgeon et al, 1994; Jhamb et al 2007; Sun et al, 2013), whereas some do not support a positive association (Piper et al, 1986; Kjaer et al, 1989; González et al, 1991; Jiang et al, 2009). A few studies specifically address the number of UTI episodes or the duration between UTI and UBC diagnosis, the latter to prevent potential misclassification. Overall, positive associations tend to weaken as the time lag between UTI and UBC increases (González et al, 1991; La Vecchia et al, 1991; Sun et al, 2013) and the number of UTIs decreases (Kantor et al, 1984; La Vecchia et al, 1991). In contrast to these findings, Jhamb et al (2007) and Jiang et al (2009) report a decreased risk of UBC with an increasing number of kidney and bladder infections with the lowest risk for more than three episodes of infection.

In this study, we analysed data of the Nijmegen Bladder Cancer Study (NBCS), one of the largest case-control studies for UBC worldwide, as to gain better insights into the role of recurrent infections of the (lower) urinary tract as a risk factor for UBC.

\section{MATERIALS AND METHODS}

Subjects and data extraction. Within the framework of the NBCS, UBC patients living in the catchment area of seven hospitals in the eastern part of the country were identified through the Netherlands Cancer Registry (NCR) held by the Comprehensive Cancer Centre the Netherlands (IKNL) (Kiemeney et al, 2008). Patients diagnosed in the periods 1995-2006, 2006-2007, 2007-2009, and 2009-2010 under the age of 76 years were batch-wise invited for participation. Participants were asked to fill out a questionnaire and donate a blood sample for DNA extraction. All patients had histologically confirmed carcinoma of the urinary bladder. Data on tumour characteristics were obtained via the NCR. The response rate to the questionnaire was $65 \%$.

Controls in the NBCS were individuals recruited for the Nijmegen Biomedical Study (NBS), a population-based survey that aimed to provide a generic reference group for case-control studies. The NBS was conducted by the Departments for Health Evidence and Clinical Chemistry of the Radboud University Medical Center, Nijmegen, The Netherlands. In 2002, 22451 ageand sex-stratified inhabitants were randomly selected from the demography registration of the municipality of Nijmegen and invited to fill out a questionnaire and donate a blood sample. The response rate to this first questionnaire was $43 \%$, with a total of 9350 participants (54\% female). A second, third, and fourth questionnaire were sent out in 2005 (response rate $70 \% ; n=5594$ ), 2008 (response rate $69 \% ; n=5613$ ), and 2012 (response rate 51\%; $n=3831$ ), respectively.

The study protocols of the NBCS and the NBS were approved by the Institutional Review Board of the Radboud University Medical Center, and all study subjects gave written informed consent (Kiemeney et al, 2008) (NBCS: http://icbc.cancer.gov/membership.html; NBS: http://www.nijmegenbiomedischestudie.nl).
By means of the NBCS and NBS questionnaire(s), information on, among others, lifestyle, ethnicity, medical history, and family history, was collected from the patients and controls. Data on UTIs were retrieved via two questions: (i) Have you ever been diagnosed by a physician with regular bladder infections (for the remainder of this article abbreviated with regular low-UTI), and, if so, at what age? (ii) Have you ever had a urinary tract infection for which you were treated with antibiotics (abbreviated with UTI-ab) and, if so, how often $(1-2,3-5,6-10$, or 11 times or more) and at what age did you first have a UTI-ab? For patients, the latter question was specifically posed for the period up to 2 years prior to UBC diagnosis. For the NBS controls, data on regular low-UTI were obtained in 2005 and data on UTI-ab were obtained in 2008. We excluded those UTI-ab in controls that were reported to have occurred within 2 years prior to filling out the questionnaire. For regular low-UTI, those that occurred at or after age at UBC diagnosis for patients (and filling out the questionnaire for controls) were excluded.

In addition, questionnaire data on highest education, ethnicity, smoking, and regular use during a minimum of 6 months of the non-steroidal anti-inflammatory drugs aspirin and ibuprofen/ brufen (prior to UBC diagnosis) were extracted.

For this study, we selected UBC patients of self-reported Caucasian ethnicity and controls that filled out questionnaires 1-3 (that contained information relevant for this study), had no prior history of bladder cancer at time of recruitment, and were of selfreported Caucasian ethnicity.

Statistical analysis. Univariable and multivariable logistic regression analyses were used to evaluate the association between regular low-UTI and frequency of UTI-ab and bladder cancer risk. Odds ratios (ORs) and corresponding 95\% confidence intervals (CIs) were adjusted for the following, potentially confounding, factors: age at UBC diagnosis/at time of completing questionnaire (in years), smoking status (categorical variable: never smokers, former smokers, current smokers (i.e., those who had not stopped in the year prior to the year of UBC diagnosis (for patients) or filling out the questionnaire (for controls)), cumulative smoking exposure in terms of pack-years of cigarettes, use of aspirin and ibuprofen/ brufen (yes/no), and education (categorical variable). Additional analyses were conducted to determine whether the associations differed for non-muscle invasive and muscle-invasive bladder cancer, age at onset of UTI (below or above sex-specific median age), menopausal state at onset UTI (using a threshold of 51 years as proxy), and smoking status (never $v s$ ever). All analyses were stratified for gender. A (two-sided) $P$-value of less than 0.05 was considered statistically significant. Statistical analyses were performed using IBM SPSS Statistics for Windows, release 20.0.0.1 (IBM Corp., Armonk, NY, USA).

\section{RESULTS}

Characteristics of UBC patients and controls. This study included 1809 UBC patients (81\% male) and 4370 controls (45\% male). Average age of male patients and controls was 63 and 62 years, respectively. Average age for female patients and controls was 61 and 57 years. Patients were less educated, were more likely to smoke, and more likely to have regularly used non-steroidal anti-inflammatory drugs, as compared with controls. Among the patients, $83 \%$ of male and $75 \%$ of female cases were categorised as non-muscle invasive bladder cancer patients (stages Ta, T1, and CIS). Almost all patients were diagnosed with urothelial carcinoma (Table 1).

The frequencies of regular low-UTI and UTI-ab in patients and controls are given in Table 2. As expected, these were higher in women compared with men. The overlap in reported regular low- 
Table 1. Baseline characteristics of the study population

\begin{tabular}{|c|c|c|c|c|}
\hline & \multicolumn{2}{|c|}{ Men $(n=3437)$} & \multicolumn{2}{|c|}{ Women $(n=2742)$} \\
\hline & $\begin{array}{l}\text { Controls } \\
(n=1974)\end{array}$ & $\begin{array}{l}\text { Patients } \\
(n=1463)\end{array}$ & $\begin{array}{l}\text { Controls } \\
(n=2396)\end{array}$ & $\begin{array}{l}\text { Patients } \\
(n=346)\end{array}$ \\
\hline Mean age in years (sd) & $61.8(15.9)$ & $62.9(9.3)$ & $56.5(16.5)$ & $61.3(10.6)$ \\
\hline \multicolumn{5}{|l|}{ Highest education } \\
\hline $\begin{array}{l}\text { Primary } \\
\text { Lower vocational secondary } \\
\text { Intermediate general secondary } \\
\text { Intermediate vocational secondary } \\
\text { Higher general secondary } \\
\text { Higher vocational secondary } \\
\text { University } \\
\text { Other }\end{array}$ & $\begin{array}{l}122(6.2) \\
305(15.5) \\
246(12.5) \\
296(15.1) \\
186(9.5) \\
379(19.3) \\
428(21.8) \\
\quad 3(0.2)\end{array}$ & $\begin{aligned} & 213(14.6) \\
& 363(24.8) \\
& 202(13.8) \\
& 219(15.0) \\
& 84(5.7) \\
& 219(15.0) \\
& 113(7.7) \\
& 49(3.4)\end{aligned}$ & $\begin{array}{c}184(7.7) \\
307(12.9) \\
432(18.1) \\
277(11.6) \\
276(11.6) \\
580(24.3) \\
331(13.9) \\
1(0.0)\end{array}$ & $\begin{aligned} 70 & (20.2) \\
82 & (23.7) \\
87 & (25.1) \\
36 & (10.4) \\
13 & (3.8) \\
39 & (11.3) \\
5 & (1.4) \\
14 & (4.0)\end{aligned}$ \\
\hline \multicolumn{5}{|l|}{ Smoking status } \\
\hline $\begin{array}{l}\text { Never } \\
\text { Former } \\
\text { Current } \\
\text { Mean pack-years cigarettes (sd) }\end{array}$ & $\begin{array}{r}483(24.5) \\
1170(59.3) \\
319(16.2) \\
19.1(17.1)\end{array}$ & $\begin{array}{l}120(8.2) \\
860(58.8) \\
482(33.0) \\
27.0(19.2)\end{array}$ & $\begin{array}{r}1034(43.3) \\
996(41.7) \\
358(15.0) \\
14.1(15.2)\end{array}$ & $\begin{array}{r}88(25.4) \\
131(37.9) \\
127(36.7) \\
22.5(16.0)\end{array}$ \\
\hline \multicolumn{5}{|l|}{ NSAIDS } \\
\hline $\begin{array}{l}\text { Aspirin } \\
\text { lbuprofen/brufen }\end{array}$ & $\begin{array}{l}474(24.0) \\
232(11.8)\end{array}$ & $\begin{array}{l}524(35.8) \\
239(16.3)\end{array}$ & $\begin{array}{l}462(19.3) \\
503(21.0)\end{array}$ & $\begin{array}{r}126(36.4) \\
73(21.1)\end{array}$ \\
\hline \multicolumn{5}{|l|}{ Tumour stage } \\
\hline $\begin{array}{l}\text { NMIBC (Ta, T1, CIS) } \\
\text { MIBC ( } \geqslant T 2) \\
\text { Missing }\end{array}$ & $\begin{array}{l}- \\
-\end{array}$ & $\begin{array}{c}1220(83.4) \\
183(12.5) \\
60(4.1)\end{array}$ & $\begin{array}{l}- \\
-\end{array}$ & $\begin{array}{r}260(75.1) \\
70(20.2) \\
16(4.6)\end{array}$ \\
\hline \multicolumn{5}{|l|}{ Tumour histology } \\
\hline $\begin{array}{l}\text { Urothelial carcinoma } \\
\text { Squamous cell carcinoma } \\
\text { Adenocarcinoma } \\
\text { Other }\end{array}$ & $\begin{array}{l}- \\
- \\
-\end{array}$ & $\begin{aligned} 1440 & (98.4) \\
4 & (0.3) \\
5 & (0.3) \\
14 & (1.0)\end{aligned}$ & $\begin{array}{l}- \\
- \\
-\end{array}$ & $\begin{aligned} 327 & (94.5) \\
11 & (3.2) \\
6 & (1.7) \\
2 & (0.6)\end{aligned}$ \\
\hline
\end{tabular}

UTI and frequent UTI-ab by the study participants was considerable (Table 3).

Association between UTI and UBC. A positive history of regular low-UTI was associated with an increased risk of UBC in both men $\left(\mathrm{OR}_{\text {adjusted }}(95 \% \mathrm{CI}) 6.6(4.2-11)\right)$ and women $\left(\mathrm{OR}_{\mathrm{adj}} 2.7\right.$ (2.0-3.5)). In contrast, a positive history of UTI-ab was associated with a decreased UBC risk, again in both men $\left(\mathrm{OR}_{\mathrm{adj}} 0.76\right.$ $(0.61-0.94))$ and women $\left(\mathrm{OR}_{\mathrm{adj}} 0.82(0.64-1.1)\right)$. Stratification on frequency of UTI-ab revealed that the protective effect was only seen for up to five episodes of infection. An increased UBC risk was observed for men with more than 10 UTI-ab episodes $\left(\mathrm{OR}_{\mathrm{adj}} 6.0\right.$ $(1.2-30))$ but numbers of male patients and controls in this stratum were low (Table 2).

As the symptoms of bladder cancer may be confused with those of a UTI, we repeated the analyses while omitting those regular low-UTI or UTI-ab that took place within 5 years prior to the date of diagnosis of bladder cancer (for patients) or the questionnaire (for controls). Resulting risks were only slightly changed (Table 2) and for subsequent analyses, we did not take the time lag between UTI and UBC into account.

The stratified analyses (Table 4) indicated that the UBC risk increase for regular low-UTI and multiple episodes of UTI-ab was more pronounced in muscle-invasive bladder cancer compared with non-muscle invasive bladder cancer. The UBC risk decrease for a low number of UTI-ab is specifically observed in those who (had) smoked and the group of subjects that experienced UTI at a younger age (i.e., below the sex-specific median age at onset UTI). For women, large differences in UTI-ab-associated risks for premenopausal ( $\leqslant 51$ years) vs postmenopausal ( $>51$ years) state were seen. The decrease of UBC risk for a limited number of UTIab was only observed for premenopausal state UTI; a strong increase in risk for UBC was observed for postmenopausal state UTI-ab, irrespective of the number of episodes. Omission of UTI$\mathrm{ab}$ that occurred within 5 years prior to UBC diagnosis/ questionnaire led to even higher risks (data not shown).

\section{DISCUSSION}

Infection of the urinary tract has been thought to play a role in urinary bladder carcinogenesis, but has only been particularly evidenced and widely accepted for the association between schistosomiasis and catheter use in spinal cord injury with squamous cell carcinoma (IARC Working Group on the Evaluation of Carcinogenic Risks to Humans, 1994; Hess et al, 2003). We show in this case-control study that UTI in the form of regular cystitis is associated with an increased risk of carcinoma of the bladder and that a limited number of UTI treated with antibiotics (i.e., $\leqslant 5$ ) is associated with decreased UBC risk.

We analysed data on regular cystitis and the number of UTI treated with antibiotics. These two variables are both measures of recurrent UTI but do not reflect the exact same information. In addition to differential recall bias or misclassification, disparity between results for regular low-UTI and a high number of UTI-ab as observed in our study can be contributed to the distinctions between (i) regular and a high number of episodes, (ii) cystitis and UTIs, and (iii) use and no use of antibiotics. Indeed, disparate risks for bladder and kidney infections have been reported by Jiang et al (2009) and Jhamb et al (2007). Also, inhibition of cell proliferation and anti-cancer effects in transitional cell carcinoma cell lines for two of the most commonly used antibiotics in UTI in Dutch 
Table 4. Associations between regular bladder infection (regular low-UTI) and UTI-ab and urinary bladder cancer, stratified by stage of bladder cancer, median age at onset of infection, menopausal state at onset infection (i.e., older than 51 years of age or not), and smoking status (never vs ever)

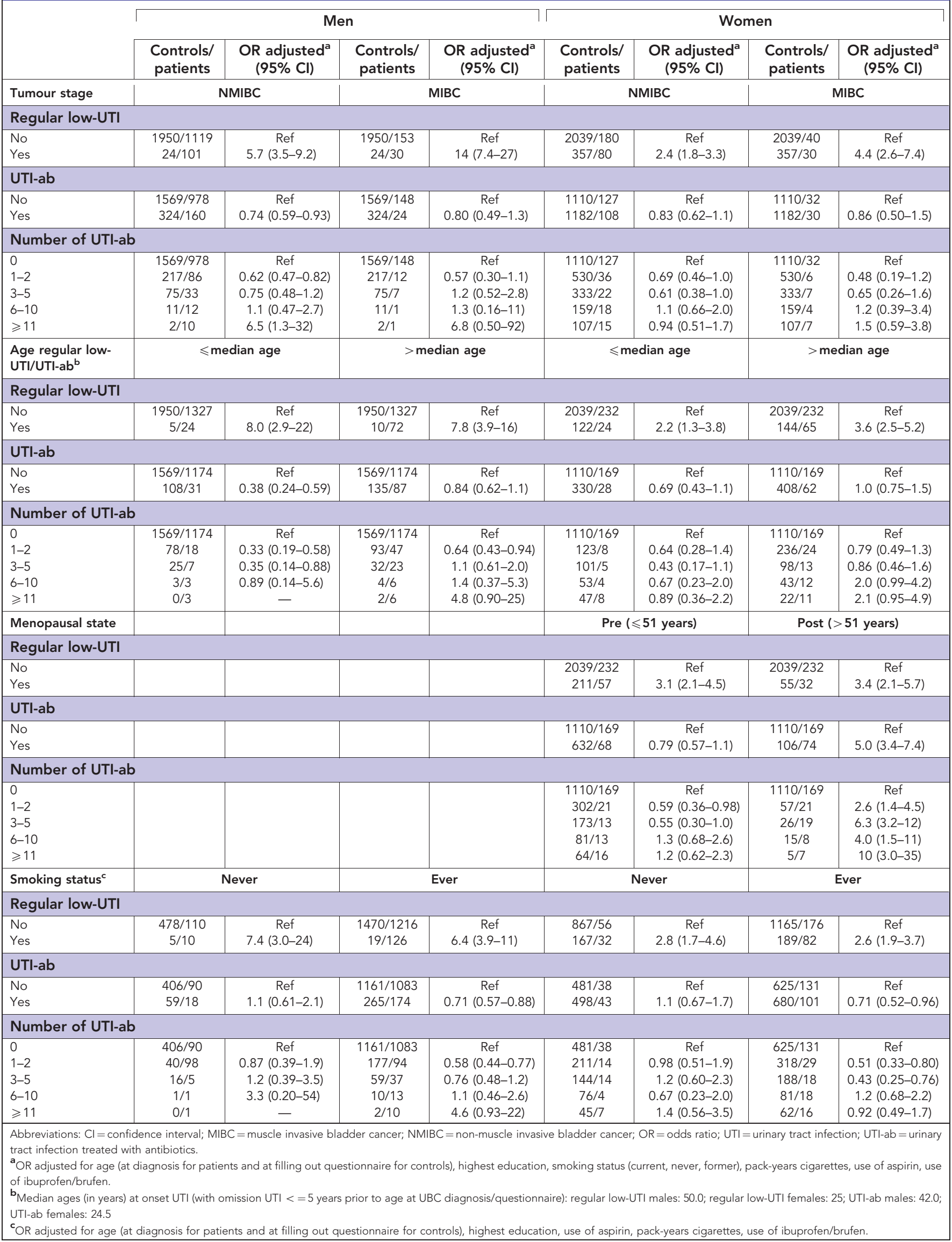


speculate that the protective effect of a limited number of UTI treated with antibiotics suggests that the beneficial anti-cancer effect of antibiotics as described by Seay et al (1996) and Kamat and Lamm (2004) outweighs the detrimental effects of (sporadic) infection and subsequent NO production. It is, however, unclear whether only a few short courses of antibiotics can decrease bladder cancer risk. Note that the decreased rate of UTI-ab in bladder cancer patients compared with controls was not seen for the few UBC patients in our population with squamous cell and adenocarcinoma but only for urothelial carcinoma (as predominant histology) (data not shown).

Exact comparison between our findings and those from previous studies is hampered by the fact that only few studies have addressed the number, location, and treatment of the UTIs in detail. The observed increased UBC risk for regular cystitis confirms findings from Kantor et al (1984) and La Vecchia et al (1991) whose studies show that more than three episodes of cystitis increases UBC risk. The observed decreased risk of UBC for a limited number of UTI is in line with a recent study from Jiang et al (2009) in which one, two to three, and more than three episodes of cystitis were associated with reduced risk of UBC in women. Unfortunately, those women who experienced more than three episodes were combined in one category, thereby preventing us to evaluate the association for those with a higher number of episodes in more detail. Most studies, however, report a higher UBC risk for a patient-reported positive history of UTI (Wynder et al, 1963; Jhamb et al, 2007).

A number of the previous studies into the association between UTI and UBC have described a decrease in the OR for UTI and bladder cancer risk after excluding those UTI that occurred within the years prior to UBC diagnosis (González et al, 1991; La Vecchia et al, 1991; Jhamb et al, 2007; Jiang et al, 2009; Sun et al, 2013). These findings support the existence of a diagnostic bias. This is underlined by studies that have described UTI diagnosis as a cause for delay in bladder cancer diagnosis (Henning et al, 2013; Cohn et al, 2014). The results of our study were, however, only marginally affected by omission of infections occurring within 5 years prior to UBC diagnosis.

We showed that our results for UTI-ab were similar for men and women; the increased UBC risk for regular cystitis was higher in men though. However, note that, in contrast to women, regular cystitis is rare among the male population. Further stratified analyses, more or less hampered by limited numbers in specific subcategories, did identify deviating findings in some subgroups. Timing of the onset of UTI was found to be of importance; the protective effect of a small number of UTI-ab was only found for those UTI that were experienced at a relatively young age (i.e., below the sex-specific median age) and the increase in UBC risk for recurrent cystitis was more pronounced for an older age of UTI-onset. Especially the increased risk of bladder cancer for history of UTI-ab, irrespective of frequency, in postmenopausal women was striking and warrants further research. It is notable that these subgroups that showed stronger increased UBC risk for recurrent cystitis, that is, men, those with UTI onset at an older age, and postmenopausal women, are also more likely to experience a complicated and recurrent (course of) UTI because of the presence of anatomical, functional, or metabolic conditions (e.g., prostatitis, diabetes, vaginal atrophy). Furthermore, the higher UBC risk for regular cystitis was much stronger in muscleinvasive bladder cancer compared with non-muscle invasive bladder cancer, in line with Kantor et al (1984) and Sturgeon et al (1994). The protective effect of a small number of UTI-ab was only seen in (former) smokers; we could not confirm the positive interaction between smoking and history of cystitis on UBC risk as reported in earlier studies (Kantor et al, 1984; La Vecchia et al, 1991).
In conclusion, our study showed a protective effect on UBC risk for a small number $(\leqslant 5)$ of UTI episodes treated with antibiotics, specifically at a younger age of onset and in (former) smokers, and increased UBC risk for regular cystitis. We underline the importance of considering the total number of UTI, age at onset, location in the urinary tract, and use of antibiotics when considering the association of UTI with bladder cancer in future research as to allow for further unravelling of their separate effects.

\section{ACKNOWLEDGEMENTS}

Principal investigators of the Nijmegen Biomedical Study are LA Kiemeney, M den Heijer, ALM Verbeek, and DW Swinkels en B Franke.

\section{REFERENCES}

Claude J, Kunze E, Frentzel-Beyme R, Paczkowski K, Schneider J, Schubert H (1986) Life-style and occupational risk factors in cancer of the lower urinary tract. Am J Epidemiol 124: 578-589.

Cohn JA, Vekhter B, Lyttle C, Steinberg GD, Large MC (2014) Sex disparities in diagnosis of bladder cancer after initial presentation with hematuria: a nationwide claims-based investigation. Cancer 120: 555-561.

Dunham LJ, Rabson AS, Stewart HL, Frank AS, Young JL (1968) Rates, interview, and pathology study of cancer of the urinary bladder in New Orleans, Louisiana. J Natl Cancer Inst 41: 683-709.

Foxman B, Gillespie B, Koopman J, Zhang L, Palin K, Tallman P, Marsh JV, Spear S, Sobel JD, Marty MJ, Marrs CF (2000) Risk factors for second urinary tract infection among college women. Am J Epidemiol 151: 1194-1205.

Foxman B (2010) The epidemiology of urinary tract infection. Nat Rev Urol 7: 653-660.

González CA, Errezola M, Izarzugaza I, López-Abente G, Escolar A, Nebot M, Riboli E (1991) Urinary infection, renal lithiasis and bladder cancer in Spain. Eur J Cancer 27: 498-500.

Henning A, Wehrberger M, Madersbacher S, Pycha A, Martini T, Comploj E, Jeschke K, Tripolt C, Rauchenwald M (2013) Do differences in clinical symptoms and referral patterns contribute to the gender gap in bladder cancer? BJU Int 112: 68-73.

Hess MJ, Zhan EH, Foo DK, Yalla SV (2003) Bladder cancer in patients with spinal cord injury. J Spinal Cord Med 26: 335-338.

Howe GR, Burch JD, Miller AB, Cook GM, Esteve G, Morrison B, Gordon P, Chambers LW, Fodor G, Winsor GM (1980) Tobacco use, occupation, coffee, various nutrients and bladder cancer. J Natl Cancer Inst 64: 701-713.

IARC Working Group on the Evaluation of Carcinogenic Risks to Humans (1994) Schistosomes, liver flukes and Helicobacter pylori. IARC Monogr Eval Carcinog Risks Hum 61: 1-241.

Jhamb M, Lin J, Ballow R, Kamat AM, Grossman HB, Wu X (2007) Urinary tract diseases and bladder cancer risk: a case-control study. Cancer Causes Control 18: 839-845.

Jiang X, Castelao JE, Groshen S, Cortessis VK, Shibata D, Conti DV, Yuan JM, Pike MC, Gago-Dominguez M (2009) Urinary tract infections and reduced risk of bladder cancer in Los Angeles. Br J Cancer 100: 834-839.

Kamat AM, Lamm DL (2004) Antitumor activity of common antibiotics against superficial bladder cancer. Urology 63: 457-460.

Kantor AF, Hartge P, Hoover RN, Narayana AS, Sullivan JW, Fraumeni Jr JF (1984) Urinary tract infection and risk of bladder cancer. Am J Epidemiol 119: $510-515$.

Kiemeney LA, Thorlacius S, Sulem P, Geller F, Aben KK, Stacey SN, Gudmundsson J, Jakobsdottir M, Bergthorsson JT, Sigurdsson A, Blondal T, Witjes JA, Vermeulen SH, Hulsbergen-van de Kaa CA, Swinkels DW, Ploeg M, Cornel EB, Vergunst H, Thorgeirsson TE, Gudbjartsson D, Gudjonsson SA, Thorleifsson G, Kristinsson KT, Mouy M, Snorradottir S, Placidi D, Campagna M, Arici C, Koppova K, Gurzau E, Rudnai P, Kellen E, Polidoro S, Guarrera S, Sacerdote C, Sanchez M, Saez B, Valdivia G, Ryk C, de Verdier P, Lindblom A, Golka K, Bishop DT, Knowles MA, Nikulasson S, Petursdottir V, Jonsson E, Geirsson G, Kristjansson B, Mayordomo JI, Steineck G, Porru S, 
Buntinx F, Zeegers MP, Fletcher T, Kumar R, Matullo G, Vineis P, Kiltie AE, Gulcher JR, Thorsteinsdottir U, Kong A, Rafnar T, Stefansson K (2008) Sequence variant on 8q24 confers susceptibility to urinary bladder cancer. Nat Genet 40: 1307-1312.

Kjaer SK, Knudsen JB, Sorensen BL, Moller Jensen O (1989) The Copenhagen case-control study of bladder cancer. V. Review of the role of urinary-tract infection. Acta Oncol 28: 631-636.

Kunze E, Chang-Claude J, Frentzel-Beyme R (1992) Life style and occupational risk factors for bladder cancer in Germany. A case-control study. Cancer 69: 1776-1790.

La Vecchia C, Negri E, D’Avanzo B, Savoldelli R, Franceschi S (1991) Genital and urinary tract diseases and bladder cancer. Cancer Res 51: 629-631.

Lin Z, Chen S, Ye C, Zhu S (2003) Nitric oxide synthase expression in human bladder cancer and its relation to angiogenesis. Urol Res 31: 232-235.

Ma N, Thanan R, Kobayashi H, Hammam O, Wishahi M, El Leithy T, Hiraku Y, Amro el-K, Oikawa S, Ohnishi S, Murata M, Kawanishi S (2011) Nitrative DNA damage and Oct3/4 expression in urinary bladder cancer with Schistosoma haematobium infection. Biochem Biophys Res Commun 414: 344-349.

Mysorekar IU, Mulvey MA, Hultgren SJ, Gordon JI (2002) Molecular regulation of urothelial renewal and host defenses during infection with uropathogenic Escherichia coli. J Biol Chem 277: 7412-7419.

Nicolle LE (2002) Epidemiology of urinary tract infections. Clin Microb Newsletter 18: 135-140.

Piper JM, Matanoski GM, Tonascia J (1986) Bladder cancer in young women. Am J Epidemiol 123: 1033-1042.
Poljakovic M, Persson K (2003) Urinary tract infection in iNOS-deficient mice with focus on bacterial sensitivity to nitric oxide. Am J Physiol Renal Physiol 284: F22-F31.

Salvatore S, Salvatore S, Cattoni E, Siesto G, Serati M, Sorice P, Torella M (2011) Urinary tract infections in women. Eur J Obstet Gynecol Reprod Biol 156: 131-136.

Seay TM, Peretsman SJ, Dixon PS (1996) Inhibition of human transitional cell carcinoma in vitro proliferation by fluoroquinolone antibiotics. J Urol 155: 757-762.

Sturgeon SR, Hartge P, Silverman DT, Kantor AF, Linehan WM, Lynch C, Hoover RN (1994) Associations between bladder cancer risk factors and tumor stage and grade at diagnosis. Epidemiology 5: 218-225.

Sun LM, Lin CL, Liang JA, Liu SH, Sung FC, Chang YJ, Kao CH (2013) Urinary tract infection increases subsequent urinary tract cancer risk: a population-based cohort study. Cancer Sci 104: 619-623.

Swana HS, Smith SD, Perrotta PL, Saito N, Wheeler MA, Weiss RM (1999) Inducible nitric oxide synthase with transitional cell carcinoma of the bladder. J Urol 161: 630-634.

Wynder EL, Onderdonk J, Mantel N (1963) An epidemiological investigation of cancer of the bladder. Cancer 16: 1388-1407.

Xu W, Liu LZ, Loizidou M, Ahmed M, Charles IG (2002) The role of nitric oxide in cancer. Cell Res 12: 311-320.

This work is published under the standard license to publish agreement. After 12 months the work will become freely available and the license terms will switch to a Creative Commons AttributionNonCommercial-Share Alike 3.0 Unported License. 